\title{
AN INEQUALITY FOR PRODUCTS OF POLYNOMIALS
}

\author{
BRUCE REZNICK
}

(Communicated by Palle E. T. Jorgensen)

\begin{abstract}
Beauzamy, Bombieri, Enflo, and Montgomery recently established an inequality for the coefficients of products of homogeneous polynomials in several variables with complex coefficients (forms). We give this inequality an alternative interpretation: let $f$ be a form of degree $m$, let $f(D)$ denote the associated $m$ th order differential operator, and define $\|f\|$ by $\|f\|^{2}=f(D) \bar{f}$. Then $\|p q\| \geq\|p\|\|q\|$ for all forms $p$ and $q$, regardless of degree or number of variables. Our principal result is that $\|p q\|=\|p\|\|q\|$ if and only if, after a unitary change of variables, $p$ and $q$ are forms in disjoint sets of variables. This is achieved via an explicit formula for $\|p q\|^{2}$ in terms of the coefficients of $p$ and $q$.
\end{abstract}

\section{INTRODUCTION}

Recently, Beauzamy, Bombieri, Enflo, and Montgomery [1, Theorem 1.2] established an inequality for the coefficients of products of homogeneous polynomials with complex coefficients (forms). They defined a norm $[p]_{2}$ on forms (see (2.13)) and showed that if $p$ and $q$ are forms in $n$ variables with degrees $d$ and $e$, respectively, then

$$
[p q]_{2} \geq \frac{(d ! e !)^{1 / 2}}{((d+e) !)^{1 / 2}}[p]_{2}[q]_{2} .
$$

They remarked that (1.1) holds independently of the number of variables. It is trivial to observe that if we set

$$
\|f\|=((\operatorname{deg} f) !)^{1 / 2}[f]_{2},
$$

then (1.1) becomes an inequality that holds for forms independently of both the number of variables and the degree:

$$
\|p q\| \geq\|p\|\|q\| \text {. }
$$

In this paper, we shall give a less contrived definition of $\|\cdot\|$ and study the case of equality in (1.3).

In $\S 2$ we discuss the vector spaces of forms of fixed degree in a fixed number of variables. For a form $f$ of degree $d$, let $f(D)$ be the associated $d$ th order

Received by the editors July 29,1991 .

1991 Mathematics Subject Classification. Primary 26D10; Secondary 15A63, 11E39, 12D05.

The author was supported in part by the National Science Foundation. 
differential operator. We show that $\|f\|^{2}=f(D) \bar{f}$, and if $f$ and $g$ are related by a unitary change of variables then $\|f\|=\|g\|$.

In $\S 3$ we prove the main new result of this paper. We say that two forms $p$ and $q$ are "unitarily disjoint" if, after a unitary (linear) change of variables, they depend on disjoint sets of variables. Equivalently, $p$ and $q$ are unitarily disjoint if there exists an orthogonal decomposition $\mathbb{C}^{n}=A \oplus B$ so that, for all $x, \nabla p(x) \in A$ and $\nabla q(x) \in B$.

Main Theorem. Let $p$ and $q$ be forms. Then $\|p q\|=\|p\|\|q\|$ if and only if $p$ and $q$ are unitarily disjoint.

Beauzamy [2] showed (using combinatorial identities) that (1.1) (and therefore (1.3) as well) is sharp for $p\left(x_{1}, x_{2}\right)=\left(x_{1}+x_{2}\right)^{d}$ and $q\left(x_{1}, x_{2}\right)=\left(x_{1}-x_{2}\right)^{d}$. The main theorem implies that the only such examples among binary forms have the shape $p\left(x_{1}, x_{2}\right)=\lambda\left(\alpha x_{1}+\beta x_{2}\right)^{d}$ and $q\left(x_{1}, x_{2}\right)=\mu\left(\bar{\beta} x_{1}-\bar{\alpha} x_{2}\right)^{e}$ for complex numbers $\lambda, \mu, \alpha$, and $\beta$ and integers $d$ and $e$. (Two of Beauzamy's students, J.-L. Frot and C. Millour (see [3]), also proved the main theorem very recently, using the multilinear approach of $[1,2]$. Their proof and ours are substantially different.)

Our proof of the main theorem relies on an explicit and complicated formula for $\|p q\|^{2}$, inspired by a reading of [1], that leads immediately to an independent proof of (1.3). We give a combinatorial proof of this product formula in $\S 4$. Section 5 contains some implications of the formula and possible areas of future work.

\section{THE VECTOR SPACE OF $n$-ARY $d$-IC FORMS}

The contents of this section are very similar to parts of [5], in which attention is restricted to real forms.

Fix $n \geq 2$ and $d \geq 1$, and let $\mathscr{F}_{n, d}$ denote the vector space of homogeneous polynomials $p\left(x_{1}, \ldots, x_{n}\right)$ of degree $d$. We write the elements of $\mathscr{F}_{n, d}$ as follows. Let $\mathscr{I}(n, d)$ denote the index set for the monomials:

$$
\mathscr{I}(n, d)=\left\{i=\left(i_{1}, \ldots, i_{n}\right): 0 \leq i_{r} \in \mathbb{Z}, \sum_{r} i_{r}=d\right\} .
$$

(Here and throughout the paper, a sum or product on $r$ shall run from 1 to $n$.) For $i \in \mathscr{I}(n, d)$ and an $n$-tuple $x$ of indeterminates, we write $x^{i}$ for $\prod_{r} x_{r}^{i_{r}}$, and for $i \in \mathscr{I}(n, d)$ we define the multinomial coefficient

$$
c(i)=d ! / \prod_{r} i_{r} !
$$

A typical element $p$ of $\mathscr{F}_{n, d}$ is written

$$
p(x)=\sum_{i \in \mathcal{I}(d)} c(i) a(p ; i) x^{i},
$$

where $a(p ; i) \in \mathbb{C}$ and $\mathscr{I}(d)$ is short for $\mathscr{I}(n, d)$ in sums, here and throughout.

We also define a complex inner product on $\mathscr{F}_{n, d}$, whose roots go back to nineteenth century projective geometry, and which has been used sporadically 
in the twentieth century (see, e.g., [4-6]). For $p, q \in \mathscr{F}_{n, d}$, let

$$
[p, q]=\sum_{i \in \mathcal{I}(d)} c(i) a(p ; i) \overline{a(q ; i)} .
$$

Among several interesting properties of $[\cdot, \cdot]$ is the way in which it is a reproducing kernel. For $\alpha \in \mathbb{C}^{n}$, define $(\alpha \cdot)^{d} \in \mathscr{F}_{n, d}$ by

$$
(\alpha \cdot)^{d}(x)=(\alpha \cdot x)^{d}=\left(\sum_{r} \alpha_{r} x_{r}\right)^{d}=\sum_{i \in \mathcal{I}(d)} c(i) \alpha^{i} x^{i}
$$

the last identity being the multinomial theorem. Then for $p \in \mathscr{F}_{n, d}$,

$$
\left[p,(\alpha \cdot)^{d}\right]=\sum_{i \in \mathcal{I}(d)} c(i) a(p ; i) \bar{\alpha}^{i}=p(\bar{\alpha}) .
$$

Every $p \in \mathscr{F}_{n, d}$ defines a $d$ th order differential operator. Let $D^{i}=\left(\partial / \partial x_{1}\right)^{i_{1}}$ $\cdots\left(\partial / \partial x_{n}\right)^{i_{n}}$ for $i \in \mathscr{I}(n, d)$, and define

$$
p(D)=\sum_{i \in \mathcal{I}(d)} c(i) a(p ; i) D^{i} .
$$

Then for $q \in \mathscr{F}_{n, d}$,

$$
p(D) \bar{q}=\sum_{i, i^{\prime} \in \mathcal{F}(d)} \sum_{c} c(i) c\left(i^{\prime}\right) a(p ; i) \overline{a\left(q ; i^{\prime}\right)} D^{i} x^{i^{\prime}} .
$$

If $i_{r}>i_{r}^{\prime}$ for any index $r$, then $D^{i} x^{i^{\prime}}=0$; since $\sum_{r} i_{r}=\sum_{r} i_{r}^{\prime}=d$, it follows that $D^{i} x^{i^{\prime}} \neq 0$ if and only if $i=i^{\prime}$. Since $D^{i} x^{i}=\prod_{r}\left(i_{r}\right) !=d ! / c(i)$, we have

$$
p(D) \bar{q}=d ! \sum_{i \in \mathcal{F}(d)} c(i) a(p ; i) \overline{a(q ; i)}=d ![p, q] .
$$

The inner product has a useful contravariant property, which is proved using a familiar folklore result. Suppose $p \in \mathscr{F}_{n, d}$ and $M$ is an $n \times n$ complex matrix. View $x$ as a column vector and define $p \circ M$ by

$$
(p \circ M)(x)=p(M x),
$$

so $\left(p \circ M_{1}\right) \circ M_{2}=p \circ\left(M_{2} M_{1}\right)$. If $p=(\alpha \cdot)^{d}$, then $p \circ M=(\alpha M \cdot)^{d}$, where $\alpha \in \mathbb{C}^{n}$ is viewed as a row vector.

Lemma 2.11 (see [5, Proposition 2.7]). For all $n$ and $d$,

$$
\mathscr{F}_{n, d}=\operatorname{span}\left\{(\alpha \cdot)^{d}: \alpha \in \mathbb{C}^{n}\right\} .
$$

Proof. If $p \in \operatorname{span}\left\{(\alpha \cdot)^{d}: \alpha \in \mathbb{C}^{n}\right\}^{\perp}$ then, by $(2.6), p(\bar{\alpha})=0$ for all $\alpha \in \mathbb{C}^{n}$, hence $p=0$.

It follows from this lemma that there exists a "basic set of nodes": $\left\{\alpha_{k}: 1 \leq\right.$ $k \leq|\mathscr{F}(n, d)|\} \subset \mathbb{C}^{n}$ so that $\left\{\left(\alpha_{k} \cdot\right)^{d}\right\}$ spans $\mathscr{F}_{n, d}$. Although we shall not need it here, $\mathscr{I}(n, d)$ itself is a basic set of nodes (see [5, Proposition 2.11]). 
Theorem 2.12 (see [5, Theorem 2.15]). Suppose $p, q \in \mathscr{F}_{n, d}$, and $M$ is a complex $n \times n$ matrix with adjoint $M^{*}$. Then $[p \circ M, q]=\left[p, q \circ M^{*}\right]$. In particular, if $M$ is unitary then $[p \circ M, q \circ M]=\left[p,(q \circ M) \circ M^{*}\right]=$ $\left[p, q \circ M^{*} M\right]=[p, q]$.

Proof. By linearity and the last lemma, it suffices to prove that $[p \circ M, q]=$ $\left[p, q \circ M^{*}\right]$ when $p$ and $q$ are $d$ th powers. By $(2.6),\left[(\alpha \cdot)^{d},(\beta \cdot)^{d}\right]=\left(\sum \alpha_{r} \beta_{r}\right)^{d}$ $=(\alpha, \beta)^{d}$, where $(\cdot, \cdot)$ is the usual $\mathbb{C}^{n}$ inner product. Thus, if $p=(\alpha \cdot)^{d}$ and $q=(\beta \cdot)^{d}$, then $[p \circ M, q]=\left[(\alpha M \cdot)^{d},(\beta \cdot)^{d}\right]=(\alpha M, \beta)^{d}=\left(\alpha, \beta M^{*}\right)^{d}=$ $\left[p, q \circ M^{*}\right]$.

This theorem can also be proved by establishing it first for $p=x^{i}$ and $q=x^{i^{\prime}}$; the resulting calculation is somewhat more involved.

In [1, Introduction] the norms $[p]_{r}, 1 \leq r<\infty$, are defined as

$$
[p]_{r}^{r}=\sum_{i \in \mathcal{I}(d)} c(i)^{1-r}|c(i) a(p ; i)|^{r}=\sum_{i \in \mathcal{I}(d)} c(i)|a(p ; i)|^{r} .
$$

We see from (2.4) and (2.13) that $[p]_{2}^{2}=[p, p]$, and from (1.2) and (2.9) that $\|p\|^{2}=D(p) \bar{p}=d ![p]_{2}^{2}$.

Corollary 2.14. If $p \in \mathscr{F}_{n, d}$ and $M$ is unitary, then $\|p\|=\|p \circ M\|$.

Proof. We have $\|p\|^{2}=d ![p, p]=d ![p \circ M, p \circ M]=\|p \circ M\|^{2}$ by Theorem 2.12 .

We conclude this section with some remarks about $p \circ M$. Suppose $M=$ $\left[m_{i j}\right]$ and let $x_{j}^{\prime}=\sum_{r} m_{j r} x_{r}$. Then, by $(2.10)$,

$$
(p \circ M)\left(x_{1}, \ldots, x_{n}\right)=p\left(x_{1}^{\prime}, \ldots, x_{n}^{\prime}\right),
$$

which is not, strictly speaking, a change of variables for $p$. However, if $M$ is unitary and $x_{j}^{*}=\sum_{r} \bar{m}_{r j} x_{r}$, then (2.15) inverts to

$$
p\left(x_{1}, \ldots, x_{n}\right)=(p \circ M)\left(x_{1}^{*}, \ldots, x_{n}^{*}\right) .
$$

\section{THE PROOF OF THE MAIN THEOREM}

We say that $p \in \mathscr{F}_{n, d}$ and $q \in \mathscr{F}_{n, e}$ are unitarily disjoint if there exists a unitary matrix $M$ and $1 \leq t \leq n$ so that $p \circ M$ is a form in $x_{1}, \ldots, x_{t}$ and $q \circ M$ is a form in $x_{t+1}, \ldots, x_{n}$. We see by (2.16) that this is equivalent to $p$ and $q$ being forms in $\left\{x_{1}^{*}, \ldots, x_{t}^{*}\right\}$ and $\left\{x_{t+1}^{*}, \ldots, x_{n}^{*}\right\}$, respectively.

Lemma 3.1. The forms $p$ and $q$ are unitarily disjoint if and only if there is an orthogonal decomposition $\mathbb{C}^{n}=A \oplus B$ such that, for all $x \in \mathbb{C}^{n}, \nabla p(x) \in A$ and $\nabla q(x) \in B$.

Proof. Suppose that $p$ and $q$ are unitarily disjoint and $M$ is the unitary matrix of the definition. For $1 \leq r \leq n$, let $D_{r}=\partial / \partial x_{r}$, so that $D_{r}(q \circ M)=0$ for $1 \leq r \leq t$ and $D_{r}(p \circ M)=0$ for $t+1 \leq r \leq n$ by hypothesis. Let $m_{r}$ denote the $r$ th column of $M$. Then (2.15) implies that $D_{r}(f \circ M)=\left(m_{r}, \overline{\nabla f}\right)$. Thus the desired decomposition of $\mathbb{C}^{n}$ occurs with $A=\operatorname{span}\left\{\bar{m}_{1}, \ldots, \bar{m}_{t}\right\}$ and $B=\operatorname{span}\left\{\bar{m}_{t+1}, \ldots \bar{m}_{n}\right\}$. Conversely, if such a decomposition exists, we may construct $M$, as above, out of orthonormal bases for $A$ and $B$.

One direction of the proof of the main theorem is now easily established. 
Theorem 3.2. Suppose $p \in \mathscr{F}_{n, d}$ and $q \in \mathscr{F}_{n, e}$ and $p$ and $q$ are unitarily disjoint. Then $\|p q\|=\|p\|\|q\|$.

Proof. By Corollary 2.14, we may assume without loss of generality that $p$ and $q$ already involve disjoint sets of variables. To emphasize this point, we write $p=p(x), q=q(y)$ and $p(D)=p\left(D_{x}\right), q(D)=q\left(D_{y}\right)$. We have

$$
p q(D)=\sum_{i \in \mathcal{I}(d)} \sum_{j \in \mathcal{I}(e)} c(i) c(j) a(p ; i) a(q ; j) D_{x}^{i} D_{y}^{j}
$$

$$
\overline{p q}=\sum_{i^{\prime} \in \mathcal{I}(d)} \sum_{j^{\prime} \in \mathcal{I}(e)} c\left(i^{\prime}\right) c\left(j^{\prime}\right) \overline{a\left(p ; i^{\prime}\right) a\left(q ; j^{\prime}\right)} x^{i^{\prime}} y^{j^{\prime}}
$$

Since the variables are disjoint, $D_{x}^{i} D_{y}^{j} x^{i^{\prime}} y^{j^{\prime}}=\left\{D_{x}^{i} x^{i^{\prime}}\right\}\left\{D_{y}^{j} y^{j^{\prime}}\right\}$, which vanishes unless $i=i^{\prime}$ and $j=j^{\prime}$, when it equals $\left\{\prod_{r}\left(i_{r}\right) !\right\}\left\{\prod_{r}\left(j_{r}\right) !\right\}$. Thus

$$
\begin{aligned}
\|p q\|^{2} & =p q(D) \overline{p q} \\
& =\sum_{i \in \mathcal{I}(d)} \sum_{j \in \mathcal{F}(e)} \prod_{r}\left(i_{r}\right) ! \prod_{r}\left(j_{r}\right) ! c(i)^{2} c(j)^{2}|a(p ; i)|^{2}|a(q ; j)|^{2} \\
& =\left\{\sum_{i \in \mathcal{F}(d)} d ! c(i)|a(p ; i)|^{2}\right\}\left\{\sum_{j \in \mathcal{F}(e)} e ! c(j)|a(q ; j)|^{2}\right\} \\
& =\|p\|^{2}\|q\|^{2} .
\end{aligned}
$$

The proof of the converse is based on Formula 3.7, which gives $\|p q\|^{2}$ in terms of $p$ and $q$; we also obtain an independent proof of (1.3). We defer the proof of Formula 3.7 to the next section.

We shall need some notation. Suppose $p \in \mathscr{F}_{n, d}$ and $q \in \mathscr{F}_{n, e}$, and suppose $0 \leq k \leq \min (d, e)$. For $\alpha \in \mathscr{I}(n, d-k)$ and $\beta \in \mathscr{I}(n, e-k)$, define

$$
A_{k}(p, q ; \alpha, \beta)=\sum_{\gamma \in \mathscr{I}(k)} c(\gamma) a(p ; \alpha+\gamma) \overline{a(q ; \beta+\gamma)} \text {. }
$$

We make some observations about $A_{k}$. If $k=0$, then $\gamma=(0, \ldots, 0)$ is the only term in the sum and $c(\gamma)=1$, so $A_{0}(p, q ; \alpha, \beta)=a(p ; \alpha) \overline{a(q ; \beta)}$. If $d=e=k$, then $\alpha$ and $\beta$ must be $(0, \ldots, 0)$ and $A_{k}(p, q ; 0,0)=[p, q]$. We shall see in Lemma 3.13 that $A_{1}(p, q ; \alpha, \beta)$ is closely related to $\nabla p$ and $\nabla q$.

Formula 3.7. Suppose $p \in \mathscr{F}_{n, d}$ and $q \in \mathscr{F}_{n, e}$. Then

$$
\|p q\|^{2}=d ! e ! \sum_{k}\left(\begin{array}{l}
d \\
k
\end{array}\right)\left(\begin{array}{l}
e \\
k
\end{array}\right) \sum_{a \in \mathcal{F}(d-k)} \sum_{\beta \in \mathcal{F}(e-k)} c(\alpha) c(\beta)\left|A_{k}(p, q ; \alpha, \beta)\right|^{2}
$$

where the sum is taken for $k \geq 0$ and $A_{k}(p ; q ; \alpha, \beta)$ is given by (3.6).

Theorem 3.9. If $p \in \mathscr{F}_{n, d}$ and $q \in \mathscr{F}_{n, e}$, then $\|p q\| \geq\|p\|\|q\|$, with equality if and only if $A_{k}(p, q ; \alpha, \beta)=0$ for all $\alpha \in \mathscr{I}(n, d-k)$ and $\beta \in \mathscr{I}(n, e-k)$ with $k \geq 1$. 
Proof. By taking the terms for $k=0$ in (3.8), we obtain the inequality

$$
\begin{aligned}
\|p q\|^{2} & \geq d ! e ! \sum_{\alpha \in \mathcal{I}(d)} \sum_{\beta \in \mathcal{F}(e)} c(\alpha) c(\beta)\left|A_{0}(p, q ; \alpha, \beta)\right|^{2} \\
& =d ! e ! \sum_{\alpha \in \mathcal{I}(d)} \sum_{\beta \in \mathcal{I}(e)} c(\alpha) c(\beta)|a(p ; \alpha)|^{2}|a(q ; \beta)|^{2}=\|p\|^{2}\|q\|^{2} .
\end{aligned}
$$

Since each $c(i)$ is positive, the remark on equality is immediate.

We turn our attention to $A_{1}$. Suppose $f \in \mathscr{F}_{n, t}$. Let $e_{1}, \ldots, e_{n}$ denote the usual unit vectors, and for $\mu \in \mathscr{I}(n, t-1)$, let

$$
\Lambda_{f ; \mu}=\left(a\left(f ; \mu+e_{1}\right), \ldots, a\left(f ; \mu+e_{n}\right)\right) \in \mathbb{C}^{n} .
$$

Since $c(\gamma)=1$ for $\gamma \in \mathscr{J}(n, 1)=\left\{e_{j}\right\}$, we may rewrite $A_{1}$ (cf. (3.6)) more succinctly in terms of the $\mathbb{C}^{n}$ inner product:

$$
A_{1}(p, q ; \alpha, \beta)=\left(\Lambda_{p ; \alpha}, \Lambda_{q ; \beta}\right) .
$$

Lemma 3.13. If $f \in \mathscr{F}_{n, t}$, then

$$
\nabla f=t \sum_{\mu \in \mathcal{I}(t-1)} c(\mu) \Lambda_{f, \mu} x^{\mu} .
$$

Proof. It suffices by symmetry to compute $\partial f / \partial x_{1}$ for

$$
\begin{gathered}
f(x)=\sum_{l \in \mathcal{I}(t)} c(l) a(f ; l) x^{l}: \\
\frac{\partial f}{\partial x_{1}}=\sum_{l \in \mathcal{I}(t)} l_{1} c(l) a(f ; l) x^{l-e_{1}} .
\end{gathered}
$$

We rewrite this sum by letting $\mu=l-e_{1} \in \mathscr{F}(n, t-1)$. Note that if $l-e_{1} \notin$ $\mathscr{I}(n, t-1)$ then $l_{1}=0$ and the term does not occur in the sum. Note also that $l_{1} c(l)=\left(\mu_{1}+1\right) c\left(\mu+e_{1}\right)=t c(\mu)$. Thus, (3.14) becomes

$$
\frac{\partial f}{\partial x_{1}}=\sum_{\mu \in \mathcal{I}(t-1)} t c(\mu) a\left(f ; \mu+e_{1}\right) x^{\mu} .
$$

Theorem 3.16. If $p \in \mathscr{F}_{n, d}$ and $q \in \mathscr{F}_{n, e}$ and $A_{1}(p, q ; \alpha, \beta)=0$ for all $\alpha \in \mathscr{I}(n, d-1)$ and $\beta \in \mathscr{I}(n, e-1)$, then $p$ and $q$ are unitarily disjoint.

Proof. For $f \in \mathscr{F}_{n, t}$, let $\Lambda_{f}=\operatorname{span}\left\{\Lambda_{f ; \mu}: \mu \in \mathcal{F}(t-1)\right\}$. By hypothesis, (3.11), and (3.12), $\left(\Lambda_{p ; \alpha}, \Lambda_{q ; \beta}\right)=0$ for all $(\alpha, \beta)$, and we may extend, if necessary, $\Lambda_{p} \subseteq A$ and $\Lambda_{q} \subseteq B$ into an orthogonal decomposition of $\mathbb{C}^{n}$. It follows by Lemma 3.1 that $p$ and $q$ are unitarily disjoint.

Proof of the main theorem. Combine Theorems 3.2, 3.9, and 3.16.

\section{THE PROOF OF FORMULA 3.7}

It seems likely that any proofs of this formula will be combinatorial, not conceptual. We discovered it by a careful counting of the terms that arise after all the shuffles in Theorem 1.B.2 of [1] but give a new proof here. 
We compute $\|p q\|^{2}=p q(D) \overline{p q}$ for $p \in \mathscr{F}_{n, d}$ and $q \in \mathscr{F}_{n, e}$. Since

$$
p(x) q(x)=\sum_{i \in \mathscr{I}(d)} \sum_{j \in \mathscr{I}(e)} c(i) c(j) a(p ; i) a(q ; j) x^{i+j},
$$

by definition, we have

$$
\|p q\|^{2}=\sum_{i, i^{\prime} \in \mathcal{I}(d)} \sum_{j, j^{\prime} \in \mathcal{I}(e)} \sum_{(i)} C\left(i, i^{\prime}, j, j^{\prime}\right) a(p ; i) a(q ; j) \overline{a\left(p ; i^{\prime}\right) a\left(q ; j^{\prime}\right)}
$$

where

$$
C\left(i, i^{\prime}, j, j^{\prime}\right)=c(i) c\left(i^{\prime}\right) c(j) c\left(j^{\prime}\right) D^{i+j} x^{i^{\prime}+j^{\prime}} .
$$

As in (2.8), $C\left(i, j, i^{\prime}, j^{\prime}\right)=0$ if $i+j \neq i^{\prime}+j^{\prime}$ and

$$
C\left(i, j, i^{\prime}, j^{\prime}\right)=c(i) c\left(i^{\prime}\right) c(j) c\left(j^{\prime}\right) \prod_{r}\left(l_{r} !\right), \quad \text { if } i+j=i^{\prime}+j^{\prime}=l .
$$

Observe that for $\alpha \in \mathscr{I}(d-k)$ and $\beta \in \mathscr{I}(e-k)$,

$$
\begin{aligned}
\left|A_{k}(p, q ; \alpha, \beta)\right|^{2} & \\
& =\left|\sum_{\gamma \in \mathscr{I}(k)} c(\gamma) a(p ; \alpha+\gamma) \overline{a(q ; \beta+\gamma)}\right|^{2} \\
& =\sum_{\gamma, \delta \in \mathcal{I}(k)} \sum c(\gamma) c(\delta) a(p ; \alpha+\gamma) \overline{a(q ; \beta+\gamma) a(p ; \alpha+\delta)} a(q ; \beta+\delta) .
\end{aligned}
$$

Thus, a typical term on the right-hand side of (3.8) is

$$
d ! e !\left(\begin{array}{l}
d \\
k
\end{array}\right)\left(\begin{array}{l}
e \\
k
\end{array}\right) c(\alpha) c(\beta) c(\gamma) c(\delta) a(p ; \alpha+\gamma) \overline{a(q ; \beta+\gamma) a(p ; \alpha+\delta)} a(q ; \beta+\delta) .
$$

A comparison with (4.2) shows that upon writing

$$
i=\alpha+\gamma, \quad j=\beta+\delta, \quad i^{\prime}=\alpha+\delta, \quad j^{\prime}=\beta+\gamma,
$$

every term in (4.6) satisfies $i+j=i^{\prime}+j^{\prime}=l$ (say). Let

$$
D\left(i, j, i^{\prime}, j^{\prime}\right):=\sum d ! e !\left(\begin{array}{l}
d \\
k
\end{array}\right)\left(\begin{array}{l}
e \\
k
\end{array}\right) c(\alpha) c(\beta) c(\gamma) c(\delta),
$$

where the sum is taken over all $(\alpha, \beta, \gamma, \delta)$ satisfying (4.7). Comparing (4.4) and (4.8), we see that Formula 3.7 is established if we can show that $C\left(i, j, i^{\prime}, j^{\prime}\right)=D\left(i, j, i^{\prime}, j^{\prime}\right)$ whenever $i+j=i^{\prime}+j^{\prime}$. We do this combinatorially.

Let $s, t, u, v$ each denote $n$-tuples, and define

$$
\begin{aligned}
F(s, t, u, v) & =\left(\sum_{r}\left(s_{r}+t_{r}\right)\left(u_{r}+v_{r}\right)\right)^{d+e} \\
& =\left(\sum_{r} s_{r} u_{r}+\sum_{r} s_{r} v_{r}+\sum_{r} t_{r} u_{r}+\sum_{r} t_{r} v_{r}\right)^{d+e} \\
& =\sum_{i, i^{\prime} \in \mathcal{I}(d)} \sum_{j, j^{\prime} \in \mathcal{I}(e)} \sum_{\left(i, i^{\prime}, j, j^{\prime}\right) s^{i} t^{j} u^{i^{\prime}} v^{j^{\prime}}}
\end{aligned}
$$


We compute $E\left(i, i^{\prime}, j, j^{\prime}\right)$ by two applications of the multinomial theorem.

First,

$$
F(s, t, u, v)=\sum_{\lambda \in \mathcal{F}(d+e)} c(\lambda) \prod_{r}\left\{\left(s_{r}+t_{r}\right)\left(u_{r}+v_{r}\right)\right\}^{\lambda_{r}} .
$$

The term in (4.10) that contains $s^{i} t^{j} u^{i^{\prime}} v^{j^{\prime}}$ occurs for $\lambda=i+j=i^{\prime}+j^{\prime}=l$. Thus, $E\left(i, i^{\prime}, j, j^{\prime}\right)$ is the coefficient of $s^{i} t^{j} u^{i^{\prime}} v^{j^{\prime}}$ in

$$
c(l) \prod_{r}\left(s_{r}+t_{r}\right)^{l_{r}}\left(u_{r}+v_{r}\right)^{l_{r}}
$$

namely,

$$
\begin{aligned}
\frac{(d+e) !}{\prod_{r} l_{r} !} \frac{\prod_{r} l_{r} !}{\prod_{r} i_{r} ! j_{r} !} \frac{\prod_{r} l_{r} !}{\prod_{r} i_{r}^{\prime} ! j_{r}^{\prime !}} & =\frac{(d+e) ! \prod_{r} l_{r} !}{(d !)^{2}(e !)^{2}} c(i) c(j) c\left(i^{\prime}\right) c\left(j^{\prime}\right) \\
& =(d+e) !(d !)^{-2}(e !)^{-2} C\left(i, i^{\prime}, j, j^{\prime}\right) .
\end{aligned}
$$

We may also expand $F(s, t, u, v)$ as a quadrinomial:

$$
\begin{array}{r}
F(s, t, u, v)=\sum_{\sigma \in \mathcal{F}(d+e, 4)} c(\sigma)\left(\sum_{r} s_{r} u_{r}\right)^{\sigma_{1}}\left(\sum_{r} s_{r} v_{r}\right)^{\sigma_{2}} \\
\times\left(\sum_{r} t_{r} u_{r}\right)^{\sigma_{3}}\left(\sum_{r} t_{r} v_{r}\right)^{\sigma_{4}} .
\end{array}
$$

The term $s^{i} t^{j} u^{i^{\prime}} v^{j^{\prime}}$ occurs in (4.13) for those $\sigma$ with $\sigma_{1}+\sigma_{2}=\sigma_{1}+\sigma_{3}=d$ and $\sigma_{2}+\sigma_{4}=\sigma_{3}+\sigma_{4}=e$; thus, $\sigma=(d-k, k, k, e-k)$ for $0 \leq k \leq \min (d, e)$. Therefore the coefficient of $s^{i} t^{j} u^{i^{\prime}} v^{j^{\prime}}$ in (4.13) is

$$
\sum_{\alpha, \beta, \gamma, \delta} c(\sigma) c(\alpha) c(\gamma) c(\delta) c(\beta)
$$

where the sum is over those $\alpha \in \mathscr{I}(n, d-k), \beta \in \mathscr{I}(n, e-k), \gamma \in \mathscr{I}(n, k)$, and $\delta \in \mathscr{F}(n, k)$, any $k$, satisfying (4.7). Since $c(\sigma)=\left(\begin{array}{l}d \\ k\end{array}\right)\left(\begin{array}{l}e \\ k\end{array}\right)\left(\begin{array}{c}d+e \\ e\end{array}\right),(4.8)$ implies that

$$
E\left(i, j, i^{\prime}, j^{\prime}\right)=(d+e) !(d !)^{-2}(e !)^{-2} C\left(i, i^{\prime}, j, j^{\prime}\right) .
$$

Comparison with (4.12) shows that $C\left(i, i^{\prime}, j, j^{\prime}\right)=D\left(i, j, i^{\prime}, j^{\prime}\right)$, and we are done.

\section{FinAl REMARKS AND OPEN QUESTIONS}

For a fixed form $f$, consider the ratio $L_{f}(p)=\|f p\|^{2} /\left(\|f\|^{2}\|p\|^{2}\right)$ as $p$ ranges over $\mathscr{F}_{n, d}$. Since $L_{f}(\lambda p)=L_{f}(p)$, we may restrict our attention to $p$ with $\|p\|=1$, hence the following quantities are well defined:

$$
\begin{gathered}
M_{n, d}(f)=\max \left\{L_{f}(p): p \in \mathscr{F}_{n, d}\right\}, \\
m_{n, d}(f)=\min \left\{L_{f}(p): p \in \mathscr{F}_{n, d}\right\} .
\end{gathered}
$$

If $n$ is larger than the dimension of the span of $\nabla f$, then $m_{n, d}(f)=1$, and $L_{f}(p)=1$ only if $p$ and $f$ involve unitarily disjoint variables. In any case,

$$
M_{n, d}(f)\|p\|^{2}\|q\|^{2} \geq\|p q\|^{2} \geq m_{n, d}(f)\|p\|^{2}\|q\|^{2} .
$$


The computation of $M_{n, d}(f)$ and $m_{n, d}(f)$ appear to be complicated in general. We discuss some simpler special cases. Suppose $f(x)=x^{l} \in \mathscr{F}_{n, t}$ where $l_{1} \geq l_{2} \geq \cdots \geq l_{n}$. If $p(x)=\sum_{i \in \mathcal{I}_{(d)}} c(i) a(p ; i) x^{i} \in \mathscr{F}_{n, d}$ then

$$
\begin{aligned}
L_{f}(p) & =\frac{\left\|\sum_{i \in \mathcal{I}(d)} c(i) a(p ; i) x^{i+l}\right\|^{2}}{\left\|x^{l}\right\|^{2}\left\|\sum_{i \in \mathcal{I}(d)} c(i) a(p ; i) x^{i}\right\|^{2}} \\
& =\frac{\sum_{i \in \mathcal{I}_{(d)}}\left\{\prod_{r}\left(i_{r}+l_{r}\right) !\right\} c(i)^{2}|a(p ; i)|^{2}}{\sum_{i \in \mathcal{I}_{(d)}}\left\{\prod_{r} l_{r} !\right\}\left\{\prod_{r}\left(i_{r} !\right)\right\} c(i)^{2}|a(p ; i)|^{2}} .
\end{aligned}
$$

It is clear from $(5.3)$ that $M_{n, d}(f)$ and $m_{n, d}(f)$ are, respectively, the maximum and minimum values of the quantity

$$
R(i, l)=\prod_{r=1}^{n} \frac{\left(i_{r}+l_{r}\right) !}{i_{r} ! l_{r} !}=\prod_{r=1}^{n}\left\{\prod_{k=1}^{l_{r}}\left(1+\frac{l_{r}}{k}\right)\right\},
$$

as $i$ ranges over $\mathscr{I}(n, d)$. It is not hard to prove that $R(i, l)$ achieves its minimum $\left(\begin{array}{c}l_{n}+d \\ d\end{array}\right)$ at $i=(0,0, \ldots, d)$, for $p(x)=x_{n}^{d}$. In particular, if $l_{n}=0$ then $m_{n, d}(f)=1$, as one would expect, since $f$ does not involve $x_{n}$. The behavior of the maximum of $R(i, l)$ is more delicate; it is achieved by taking the $d$ largest numbers of the form $1+l_{r} / k$. Roughly speaking, this means taking $i$ as close to $(d / m) l$ as is possible in $\mathscr{I}(n, d)$. We obtain the asymptotic estimate $M_{n, d}(f) \approx C \cdot(e d / m+o(1))^{d}$ by Stirling's formula. (Here, $e \approx 2.718$ is Euler's constant, not a degree.)

If $f \in \mathscr{F}_{2,2}$ then after a unitary change of variables, we may assume that $f\left(x_{1}, x_{2}\right)=\lambda\left\{(\cos \alpha) x_{1}^{2}-(\sin \alpha) x_{2}^{2}\right\}:=\lambda f_{\alpha}(x)$. We can show in this case that $M_{2,2}\left(f_{\alpha}\right)$ and $m_{2,2}\left(f_{\alpha}\right)$ are the two roots of the quadratic

$$
\Phi_{\alpha}(t)=t^{2}-7 t+6\left(1+\sin ^{2} 2 \alpha\right)
$$

This can be checked against the previous paragraph. If $l=(2,0)$ then $x^{l}=$ $x_{1}^{2}=f_{0}$, and the roots of $\Phi_{0}$ are 1 and 6 . It is easy to check that $R(i, l)$ equals 1 for $i=(0,2)$ and 6 for $i=(2,0)$. If $l=(1,1)$ then $x^{l}=x_{1} x_{2}$, which is unitarily equivalent to $f_{\pi / 4}$; the roots of $\Phi_{\pi / 4}$ are 3 and 4 . We see from (5.4) that $R(i, l)=3$ for $i=(2,0)$ or $(0,2)$ and $R(l, l)=4$. One might expect that, just as $L_{f}(p)=m_{n, d}(f)$ when $f$ and $p$ involve disjoint variables, then $L_{f}(p)=M_{n, d}(f)$ when $f$ and $p$ use variables in as "similar" a way as possible. The evidence given above strongly suggests that $p$ should be as close to a power of $f$ as possible; in particular, if $m=d$ then $p$ should be $f$. This evidence is misleading: we can show that $L_{f_{\alpha}}\left(f_{\alpha}\right)=M_{2,2}\left(f_{\alpha}\right)$ only when $\alpha=k \pi / 4$.

This discussion raises some obvious questions. What are the ranges of $M_{n, d}(f)$ and $m_{n, d}(f)$ as $f$ runs over $\mathscr{F}_{n, e}$ ? Clearly, the minimum value of $m_{n, d}(f)=1$; it is not hard to show that the maximum of $M_{n, d}(f)$ is $\left(\begin{array}{c}d+e \\ e\end{array}\right)$, see (5.10) below. The range of $M_{n, d}(f) / m_{n, d}(f)$ is also unknown.

We close this paper with two applications of the formula. Suppose

$$
p(x, y)=\prod_{k=1}^{d}\left\{\left(\cos \alpha_{k}\right) x-\left(\sin \alpha_{k}\right) y\right\} \in \mathscr{F}_{2, d}
$$


is a real binary form. Then a natural generalization of Leibniz's rule to the $d$ th derivative of a product of $d$ terms, whose exact statement and proof we omit, gives a closed form for $\|p\|^{2}$ :

$$
\|p\|^{2}=p(D) p=\sum_{\sigma} \prod_{k=1}^{d} \cos \left(\alpha_{k}-\alpha_{\sigma(k)}\right),
$$

where the sum in (5.7) is taken over all permutations $\sigma$ of $\{1, \ldots, d\}$. In particuiar, the sum in (5.7) is always positive.

Finally, it is easy to compute $\left\|(\alpha \cdot)^{d}\right\|^{2}$ from (2.6) and (2.9):

$$
\left\|(\alpha \cdot)^{d}\right\|^{2}=d !\left[(\alpha \cdot)^{d},(\alpha \cdot)^{d}\right]=d !(\alpha, \alpha)^{d} .
$$

This begs the natural question. If $p=(\alpha \cdot)^{d}$ and $q=(\beta \cdot)^{e}$, how does $\|p q\|$ depend on $\alpha$ and $\beta$ ? There is a satisfying geometric answer.

Corollary 5.9. If $p=(\zeta \cdot)^{d}, q=(\xi \cdot)^{e}$, and $\tau=(\zeta, \xi) /(\|\zeta\| \cdot\|\xi\|)$, then

$$
\|p q\|^{2}=\|p\|^{2}\|q\|^{2}\left(\sum_{k}\left(\begin{array}{l}
d \\
k
\end{array}\right)\left(\begin{array}{l}
e \\
k
\end{array}\right)|\tau|^{2 k}\right) .
$$

Proof. Since $a(p ; i)=\zeta^{i}$ and $a(q ; j)=\xi^{j}$, the multinomial theorem implies

$$
A_{k}(p, q ; \alpha, \beta)=\sum_{\gamma \in \mathcal{I}(k)} c(\gamma) a(p ; \alpha+\gamma) \overline{a(q ; \beta+\gamma)}=\zeta^{\alpha} \bar{\xi}^{\beta}(\zeta, \xi)^{k},
$$

and so by Formula 3.7,

$$
\|p q\|^{2}=d ! e ! \sum_{k}\left(\begin{array}{l}
d \\
k
\end{array}\right)\left(\begin{array}{l}
e \\
k
\end{array}\right)\left|(\zeta, \xi)^{k}\right|^{2} \sum_{\alpha \in \mathcal{F}(d-k)} \sum_{\beta \in \mathcal{I}(e-k)} c(\alpha) c(\beta)\left|\zeta^{\alpha} \bar{\xi}^{\beta}\right|^{2}
$$

Another application of the multinomial theorem shows that the inner sum in $(5.12)$ is $|\zeta|^{2(d-k)}|\bar{\xi}|^{2(e-k)}=(\zeta, \zeta)^{d-k}(\xi, \xi)^{e-k}$. Therefore,

$$
\|p q\|^{2}=d ! e ! \sum_{k}\left(\begin{array}{l}
d \\
k
\end{array}\right)\left(\begin{array}{l}
e \\
k
\end{array}\right)|(\zeta, \xi)|^{2 k}(\zeta, \zeta)^{d-k}(\xi, \xi)^{e-k} .
$$

An appeal to (5.8) completes the proof.

This corollary can also be proved by restricting without loss of generality to $(\zeta, \zeta)=(\xi, \xi)=1$, applying Theorem 2.12 with $M$ chosen so that $p \circ M=x_{1}^{d}$, and then using the definition of the inner product.

\section{REFERENCES}

1. B. Beauzamy, E. Bombieri, P. Enflo, and H. L. Montgomery, Products of polynomials in many variables, J. Number Theory 36 (1990), 219-245.

2. B. Beauzamy, Products of polynomials and a priori estimates for coefficients in polynomial decompositions: a sharp result, J. Symbolic Comput. (to appear).

3. B. Beauzamy, J.-L. Frot, and C. Millour, Massively parallel computations on many variable polynomials. II (in preparation).

4. S. Helgason, Groups and geometric analysis, Academic Press, New York, 1984. 
5. B. Reznick, Sums of even powers of real linear forms, Mem. Amer. Math. Soc., vol. 196, no. 463, Amer. Math. Soc., Providence, RI, 1992.

6. E. M. Stein and G. Weiss, Introduction to Fourier analysis on Euclidean spaces, Princeton Univ. Press, Princeton, NJ, 1971.

Deartment of Mathematics, University of Illinois, 1409 West Green Street, Urbana, ILLINOIS 61801

E-mail address: reznick@symcom.math.uiuc.edu 